Article

\title{
Disturbance Observer-Based Offset-Free Global Tracking Control for Input-Constrained LTI Systems with DC/DC Buck Converter Applications
}

\author{
Kyunghwan Choi ${ }^{1}\left(\mathbb{D}\right.$, Dong Soo Kim ${ }^{2}$ and Seok-Kyoon Kim ${ }^{2, *(D)}$ \\ 1 Center for Eco-Friendly \& Smart Vehicles, Korea Advanced Institute of Science and Technology, \\ Daejeon 34141, Korea; ckhwan1206@kaist.ac.kr \\ 2 Department of Creative Convergence Engineering, Hanbat National University, Daejeon 34158, Korea; \\ kds671@hanbat.ac.kr \\ * Correspondence: skkim77@hanbat.ac.kr; Tel.: +82-042-828-8801
}

Received: 25 June 2020; Accepted: 4 August 2020; Published: 6 August 2020

check for updates

\begin{abstract}
This paper presents an offset-free global tracking control algorithm for the input-constrained plants modeled as controllable and open-loop strictly stable linear time invariant (LTI) systems. The contribution of this study is two-fold: First, a global tracking control law is devised in such a way that it not only leads to offset-free reference tracking but also handles the input constraints using the invariance property of a projection operator embedded in the proposed disturbance observer (DOB). Second, the offset-free tracking property is guaranteed against uncertainties caused by plant-model mismatch using the DOB's integral action for the state estimation error. Simulation results are given in order to demonstrate the effectiveness of the proposed method by applying it to a DC/DC buck converter.
\end{abstract}

Keywords: offset-free global tracking control; input constraints; disturbance observer; LTI systems; DC/DC buck converters

\section{Introduction}

Although stabilization of dynamic systems is one of main objectives of feedback control design, output reference tracking is much more important in practice. In addition, since most control systems are subject to input constraints, it is essential to design a feedback control scheme for output reference tracking of input-constrained systems. The objective of this paper is to develop a disturbance observer (DOB) based feedback control scheme for offset-free output reference tracking of stable linear systems with input constraints and model uncertainties. If the control objective is to stabilize the system, the problem is easy to solve since zero input is a feasible stabilizing control owing to the open loop stability of the system. However, if the feedback control aims to achieve the offset-free tracking for the uncertain input-constrained system, the problem is not trivial [1-5].

Robust tracking MPC (Model Predictive Control) can be a candidate solution to the problem [6-10] since it is effective to deal with the input constraints and also capable of handling model uncertainties to some extent. On the other hand, proper computational power has to be supported in order to implement the MPC. If it is required to design a feedback control without asking computational power, a low gain feedback control can be an alternative solution [11-16]. However, since the low gain controls are devised mainly for stabilization, an integral action needs to be added on top of the low gain feedback control. In other words, in order to apply the low gain control to the output tracking problem, the tracking error dynamics between the original state equation and its desired steady state equation is computed first, and the integrator dynamics is augmented with the error dynamics. As the last step, the low gain control is designed for the augmented system. If there are no 
model uncertainties, the small gain control with the integral action can solve the tracking problem, but it can suffer from both the steady state tracking error and fulfilling the input constraints if there is plant-model mismatch. In addition to the MPC and low gain control approaches, there have been attempts to solve the tracking problem under consideration [17-19].

In order to design a robust feedback control scheme for reference tracking of stable linear systems with input constraints and model uncertainties, we propose a feedback control equipped with a DOB. The proposed DOB consists of a state observer estimating the system state and a projection operator which helps identify the steady state of the uncertain system and meet the input constraints. Thanks to the steady state of the uncertain system identified by the proposed DOB, the feedback control can achieve offset-free output reference tracking by stabilizing the tracking error dynamics, which takes the identified steady state into account. Besides, the projection operator embedded in the DOB makes the steady state control input satisfy the input constraints.

Compared with existing results in the literature, the proposed scheme has several advantages: (a) the proposed control scheme can be implemented without heavy computational power, although the input constraints are satisfied; (b) in terms of stability, the closed loop system by the proposed control results in constrained global asymptotic tracking by restricting the initial conditions of the DOB; and (c) offset-free output tracking is achieved with an improved disturbance rejection performance, even for systems with model uncertainties owing to the proposed DOB. Especially, the first and second contributions correspond to the sharp contrast with the existing results, such as input-constrained MPCs and integral controls. Constrained global asymptotic tracking means that, for an admissible reference, the offset-free tracking is achieved, fulfilling the input constraints with the plant state in $\mathbb{R}^{n}$ and the controller state in a bounded set. Note that, although the system under consideration is open loop stable, global stability is only possible when there is only one integrator, due to the input constraints. In order to demonstrate performance of the proposed control, the simulation study is done for reference tracking of a DC/DC buck converter, despite the input constraints and the load variations.

\section{Problem Formulation}

Consider the LTI systems described by

$$
\begin{aligned}
\dot{\mathbf{x}}(t) & =\mathbf{A x}(t)+\mathbf{B u}(t)+\mathbf{d}, \\
\mathbf{y}(t) & =\mathbf{C x}(t), \forall t,
\end{aligned}
$$

where $\mathbf{x}(t) \in \mathbb{R}^{n}, \mathbf{y}(t) \in \mathbb{R}^{m}$, and $\mathbf{u}(t) \in \mathbb{R}^{m}$ denote the state, output, and control input, respectively, $\mathbf{d} \in \Omega^{o} \subset \mathbb{R}^{n}$ is an unknown constant, and $\Omega^{o}$ is the interior of a known convex set $\Omega$ defined in Section 3.1. For this system, the following assumptions are made:

\section{Assumption 1.}

1. The matrices $\mathbf{A} \in \mathbb{R}^{n \times n}$ and $\mathbf{B} \in \mathbb{R}^{n \times m}$ are known.

2. The output matrix $\mathbf{C} \in \mathbb{R}^{m \times n}$ is in the form of either

$$
\mathbf{C}=\left[\begin{array}{ll}
\mathbf{0}_{m \times(n-m)} & \mathbf{I}_{m \times m}
\end{array}\right]
$$

or

$$
\mathbf{C}=\left[\begin{array}{ll}
\mathbf{I}_{m \times m} & \mathbf{0}_{m \times(n-m)}
\end{array}\right] .
$$

3. The system matrix $\mathbf{A}$ is strictly stable, i.e., all eigenvalues of the matrix $\mathbf{A}$ lie in the open left half plane.

4. The pair $(\mathbf{A}, \mathbf{B})$ is controllable.

5. The dimensions of the input $\mathbf{u}(t)$ and the output $\mathbf{y}(t)$ are the same. 
6. The control input must satisfy the input constraints, i.e.,

$$
\mathbf{u}(t) \in U:=\{\mathbf{u} \mid \underline{\mathbf{u}} \preceq \mathbf{u} \preceq \overline{\mathbf{u}}\}, \forall t
$$

where, for any $\mathbf{x}, \mathbf{y} \in \mathbb{R}^{n}, \mathbf{x} \preceq \mathbf{y}$ is defined as

$$
x_{i} \leq y_{i}, i=1,2, \cdots, m,
$$

and $x_{i}$ and $y_{i}$ denote the ith element of $\mathbf{x}$ and $\mathbf{y}$, respectively.

Definition 1. Constrained global asymptotic output tracking is said to be achieved if the controller results in the following properties:

C1. It holds that

$$
\lim _{t \rightarrow \infty} \mathbf{y}(t)=\mathbf{r}, \forall \mathbf{x}(0) \in \mathbb{R}^{n}
$$

for a given output reference signal $\mathbf{r}$.

C2. The state of the closed loop system is always bounded.

C3. The input constraints (5) is satisfied for all time.

The objective of the paper was to develop a DOB-based controller achieving the constrained global asymptotic output tracking for system (1)-(2) under Assumption 1.

Remark 1. This paper is only concerned with the case (3) because the controller design procedure is the same as the case of (4).

Remark 2. Since system (1) is open loop asymptotically stable, it is likely to think that the problem is easy to solve, even under the input constraints. This is true only when the control problem is a stabilization problem. Since this paper is concerned with an offset-free tracking problem, it is not so easy. Besides, in order to solve the tracking problem under consideration, one makes an attempt to design the Luenberger observer for the following augmented system:

$$
\left[\begin{array}{c}
\dot{\boldsymbol{x}}(t) \\
\dot{\mathbf{d}}
\end{array}\right]=\left[\begin{array}{cc}
\mathbf{A} & \mathbf{I}_{n \times n} \\
\mathbf{0}_{n \times n} & \mathbf{0}_{n \times n}
\end{array}\right]\left[\begin{array}{c}
\mathbf{x}(t) \\
\mathbf{d}
\end{array}\right]+\left[\begin{array}{c}
\mathbf{B} \\
\mathbf{0}_{n \times m}
\end{array}\right] \mathbf{u}(t), \mathbf{y}(t)=\left[\begin{array}{ll}
\mathbf{C} & \mathbf{0}_{m \times n}
\end{array}\right]\left[\begin{array}{c}
\mathbf{x}(t) \\
\mathbf{d}(t)
\end{array}\right]
$$

and a feedback law based on the estimated state if there are no input constraints and no model uncertainties. However, if there are input constraints and even model uncertainties, then the attempt suffers from the steady state error in general.

\section{Controller Law}

This section presents an offset-free constrained global tracking controller by combining the classical state-feedback controller with a DOB equipped with the projection operator. To this end, Section 3.1 derives the steady state control law for the tracking error dynamics reflecting the tracking control objective. Section 4.2 devises a state-feedback controller including a switching feedback gain and projection operator-based DOB in order to force the tracking errors to be globally convergent in the presence of input constraints. 


\subsection{Desired Steady State Control for Constrained Output Tracking}

Considering the system model and structure of the output matrix $\mathbf{C}$ in (3), the constrained output tracking problem (6) is tackled if we can solve the corresponding state tracking problem defined by

$$
\lim _{t \rightarrow \infty} \mathbf{x}(t)=\mathbf{x}^{0}(\mathbf{r}, \mathbf{d}), \forall \mathbf{x}(0) \in \mathbb{R}^{n}
$$

where $\mathbf{x}^{0}(\mathbf{r}, \mathbf{d})$ denotes the desired steady state which is a solution of the the steady state equation of the state Equation (2). In other words, it is a solution of the equation

$$
\begin{aligned}
\mathbf{0} & =\mathbf{A} \mathbf{x}^{0}(\mathbf{r}, \mathbf{d})+\mathbf{B} \mathbf{u}^{0}(\mathbf{r}, \mathbf{d})+\mathbf{d} \\
& =\left[\begin{array}{ll}
\mathbf{A}_{11} & \mathbf{A}_{12} \\
\mathbf{A}_{21} & \mathbf{A}_{22}
\end{array}\right]\left[\begin{array}{c}
\mathbf{x}_{1}^{0}(\mathbf{r}, \mathbf{d}) \\
\mathbf{r}
\end{array}\right]+\left[\begin{array}{l}
\mathbf{B}_{1} \\
\mathbf{B}_{2}
\end{array}\right] \mathbf{u}^{0}(\mathbf{r}, \mathbf{d})+\mathbf{d},
\end{aligned}
$$

where $\mathbf{A}_{11} \in \mathbb{R}^{(n-m) \times(n-m)}, \mathbf{A}_{12} \in \mathbb{R}^{(n-m) \times m}, \mathbf{A}_{21} \in \mathbb{R}^{m \times(n-m)}, \mathbf{A}_{22} \in \mathbb{R}^{m \times m}, \mathbf{B}_{1} \in \mathbb{R}^{(n-m) \times m}$, $\mathbf{B}_{2} \in \mathbb{R}^{m \times m}$, and $\mathbf{u}^{0}(\mathbf{r}, \mathbf{d})$ denotes the steady state value of the control input $\mathbf{u}(t)$. The steady state can be written as

$$
\mathbf{x}^{0}(\mathbf{r}, \mathbf{d})=\left[\begin{array}{c}
\mathbf{x}_{1}^{0}(\mathbf{r}, \mathbf{d}) \\
\mathbf{x}_{2}^{0}(\mathbf{r}, \mathbf{d})
\end{array}\right]=\left[\begin{array}{c}
\mathbf{x}_{1}^{0}(\mathbf{r}, \mathbf{d}) \\
\mathbf{y}^{0}
\end{array}\right]=\left[\begin{array}{c}
\mathbf{x}_{1}^{0}(\mathbf{r}, \mathbf{d}) \\
\mathbf{r}
\end{array}\right], \mathbf{x}_{1}^{0}(\mathbf{r}, \mathbf{d}) \in \mathbb{R}^{n-m}, \mathbf{x}_{2}^{0}(\mathbf{r}, \mathbf{d}) \in \mathbb{R}^{m},
$$

and $\mathbf{y}^{0}$ denotes the steady state value of the output $\mathbf{y}(t)$ under the input constraint (5). Furthermore, the steady state equation can be equivalently rewritten as

$$
\begin{aligned}
{\left[\begin{array}{l}
\mathbf{x}_{1}^{0}(\mathbf{r}, \mathbf{d}) \\
\mathbf{u}^{0}(\mathbf{r}, \mathbf{d})
\end{array}\right] } & =-\left[\begin{array}{ll}
\mathbf{A}_{11} & \mathbf{B}_{1} \\
\mathbf{A}_{21} & \mathbf{B}_{2}
\end{array}\right]^{-1}\left(\left[\begin{array}{l}
\mathbf{A}_{12} \\
\mathbf{A}_{22}
\end{array}\right] \mathbf{r}+\mathbf{d}\right) \\
& =:\left[\begin{array}{ll}
\mathbf{Z}_{11} & \mathbf{Z}_{12} \\
\mathbf{Z}_{21} & \mathbf{Z}_{22}
\end{array}\right]\left(\left[\begin{array}{l}
\mathbf{A}_{12} \\
\mathbf{A}_{22}
\end{array}\right] \mathbf{r}+\mathbf{d}\right),
\end{aligned}
$$

if the matrix $\left[\begin{array}{ll}\mathbf{A}_{11} & \mathbf{B}_{1} \\ \mathbf{A}_{21} & \mathbf{B}_{2}\end{array}\right]^{-1}$ exists, where the matrices $\mathbf{Z}_{i j}, i, j=1,2$, can be obtained by using the matrix inversion lemma [20] as

$$
\begin{aligned}
& \mathbf{Z}_{11}=-\mathbf{A}_{11}+\mathbf{B}_{1} \mathbf{B}_{2}^{-1} \mathbf{A}_{21} \\
& \mathbf{Z}_{12}=\left(\mathbf{A}_{11}-\mathbf{B}_{1} \mathbf{B}_{2}^{-1} \mathbf{A}_{21}\right)^{-1} \mathbf{B}_{1} \mathbf{B}_{2}^{-1}, \\
& \mathbf{Z}_{21}=\mathbf{B}_{2}^{-1} \mathbf{A}_{21}\left(\mathbf{A}_{11}-\mathbf{B}_{1} \mathbf{B}_{2}^{-1} \mathbf{A}_{21}\right)^{-1}, \\
& \mathbf{Z}_{22}=-\mathbf{B}_{2}^{-1}-\mathbf{B}_{2}^{-1} \mathbf{A}_{21}\left(\mathbf{A}_{11}-\mathbf{B}_{1} \mathbf{B}_{2}^{-1} \mathbf{A}_{21}\right)^{-1} \mathbf{B}_{1} \mathbf{B}_{2}^{-1} .
\end{aligned}
$$

The relation (10) allows the steady state control $\mathbf{u}^{0}(\mathbf{r}, \mathbf{d})$ to be expressed as in the analytic form:

$$
\mathbf{u}^{0}(\mathbf{r}, \mathbf{d})=\mathbf{D r}+\mathbf{Z}_{2} \mathbf{d}
$$

where

$$
\mathbf{D}:=\left(\mathbf{Z}_{21} \mathbf{A}_{12}+\mathbf{Z}_{22} \mathbf{A}_{22}\right), \mathbf{Z}_{2}:=\left[\begin{array}{ll}
\mathbf{Z}_{21} & \mathbf{Z}_{22}
\end{array}\right] .
$$

For the rest of the paper, it is assumed that the matrix $\left[\begin{array}{ll}\mathbf{A}_{11} & \mathbf{B}_{1} \\ \mathbf{A}_{21} & \mathbf{B}_{2}\end{array}\right]$ is invertible for the existence of the steady state control $\mathbf{u}^{0}(\mathbf{r}, \mathbf{d})$. Note that, in order to satisfy the input constraint condition (5), it is required that $\mathbf{u}^{0}(\mathbf{r}, \mathbf{d}) \in U$. In this study, a stronger assumption is made: 


\section{Assumption 2.}

$$
\mathbf{u}^{0}(\mathbf{r}, \mathbf{d}) \in U_{c} \subseteq U
$$

where $U_{c}$ is the largest circle contained in the input constraint set $U$ with the center point $\mathbf{u}_{c}$ and the radius $r_{c}$, i.e.,

$$
U_{c}=\left\{\mathbf{u} \in \mathbb{R}^{m} \mid\left\|\mathbf{u}-\mathbf{u}_{c}\right\| \leq r_{\mathcal{c}}\right\} \subseteq U
$$

It follows from Assumption 2 that the unknown constant $\mathbf{d}$ described in Section 2 is contained the interior $\Omega^{o}$ of the convex set $\Omega$ defined by

$$
\Omega:=\left\{\mathbf{d} \in \mathbb{R}^{n} \mid f(\mathbf{r}, \mathbf{d}) \leq 0\right\}
$$

with the convex function $f: \mathbb{R}^{m} \times \mathbb{R}^{n} \rightarrow \mathbb{R}$ given by

$$
\begin{aligned}
f:(\mathbf{r}, \mathbf{d}) \mapsto f(\mathbf{r}, \mathbf{d}) & :=\left\|\mathbf{D r}+\mathbf{Z}_{2} \mathbf{d}-\mathbf{u}_{c}\right\|^{2}-r_{c}^{2} \\
& =\left\|\mathbf{u}^{0}(\mathbf{r}, \mathbf{d})-\mathbf{u}_{c}\right\|^{2}-r_{c}^{2}
\end{aligned}
$$

for a given reference $\mathbf{r}$. Therefore, it reveals that, since the constant $\mathbf{d}$ is unknown, it is reasonable to consider the reference signals $\mathbf{r}$ making the steady state control $\mathbf{u}^{0}(\mathbf{r}, \mathbf{d})$ feasible, i.e., $\mathbf{u}^{0}(\mathbf{r}, \mathbf{d}) \in$ $U_{c}$. The reference $\mathbf{r}$ is called admissible when it renders the steady state control $\mathbf{u}^{0}(\mathbf{r}, \mathbf{d})$ to satisfy Assumption 2 for the rest of this paper.

\subsection{Control Law}

For the purpose of achieving offset-free global tracking, this section designs state feedback controller equipped with the DOB considering the input constraints (5). To this end, consider the steady state Equation (10) with $\mathbf{d}=\hat{\mathbf{d}}(t)$

$$
\begin{aligned}
{\left[\begin{array}{c}
\hat{\mathbf{x}}_{1}^{0}(\mathbf{r}, t) \\
\hat{\mathbf{u}}^{0}(\mathbf{r}, t)
\end{array}\right] } & :=\left.\left[\begin{array}{l}
\mathbf{x}_{1}^{0}(\mathbf{r}, \mathbf{d}) \\
\mathbf{u}^{0}(\mathbf{r}, \mathbf{d})
\end{array}\right]\right|_{\mathbf{d}=\hat{\mathbf{d}}(t)} \\
& =\left[\begin{array}{ll}
\mathbf{Z}_{11} & \mathbf{Z}_{12} \\
\mathbf{Z}_{21} & \mathbf{Z}_{22}
\end{array}\right]\left[\begin{array}{l}
\mathbf{A}_{12} \\
\mathbf{A}_{22}
\end{array}\right] \mathbf{r}+\left[\begin{array}{ll}
\mathbf{Z}_{11} & \mathbf{Z}_{12} \\
\mathbf{Z}_{21} & \mathbf{Z}_{22}
\end{array}\right] \hat{\mathbf{d}}(t), \forall t,
\end{aligned}
$$

in which another expression is given by

$$
\left[\begin{array}{c}
\hat{\mathbf{x}}_{1}^{0}(\mathbf{r}, t) \\
\hat{\mathbf{u}}^{0}(\mathbf{r}, t)
\end{array}\right]=\left[\begin{array}{l}
\mathbf{x}_{1}^{0}(\mathbf{r}, \mathbf{d}) \\
\mathbf{u}^{0}(\mathbf{r}, \mathbf{d})
\end{array}\right]-\left[\begin{array}{ll}
\mathbf{Z}_{11} & \mathbf{Z}_{12} \\
\mathbf{Z}_{21} & \mathbf{Z}_{22}
\end{array}\right] \mathbf{e}_{d}(t), \forall t,
$$

where the estimated disturbance $\hat{\mathbf{d}}(t)$ comes from the full-order DOB given by

$$
\begin{aligned}
& \dot{\hat{\mathbf{x}}}(t)=\mathbf{A x}(t)+\mathbf{B u}(t)+\hat{\mathbf{d}}(t)+\mathbf{L}_{1} \mathbf{e}_{o}(t), \\
& \dot{\hat{\mathbf{d}}}(t)=\operatorname{Proj}\left(\mathbf{L}_{2} \mathbf{e}_{o}(t)\right), \forall t,
\end{aligned}
$$


with $\hat{\mathbf{x}}(t)$ and $\hat{\mathbf{d}}(t)$ representing the estimates of the state $\mathbf{x}(t)$ and the unknown constant $\mathbf{d}$, respectively. The state estimation error $\mathbf{e}_{o}(t)$ is defined as $\mathbf{e}_{o}(t):=\mathbf{x}(t)-\hat{\mathbf{x}}(t), \forall t, \mathbf{L}_{i}=\mathbf{L}_{i}^{T}>\mathbf{0}, i=1,2$, represent the DOB gain matrices, and Proj denotes the projection operator [21] defined by

$$
\operatorname{Proj}\left(\mathbf{L}_{2} \mathbf{e}_{o}(t)\right):=\left\{\begin{array}{l}
\mathbf{L}_{2} \mathbf{e}_{o}(t), \quad \text { if } \hat{\mathbf{d}}(t) \in \Omega^{o} \text { or } \nabla f^{T} \mathbf{L}_{2} \mathbf{e}_{o}(t) \leq 0, \\
\mathbf{L}_{2} \mathbf{e}_{o}(t)-\mathbf{L}_{2} \frac{\nabla f \nabla f^{T}}{\nabla f^{T} \mathbf{L}_{2} \nabla f} \mathbf{L}_{2} \mathbf{e}_{o}(t), \text { if } \hat{\mathbf{d}}(t) \in \partial \Omega \text { and } \nabla f^{T} \mathbf{L}_{2} \mathbf{e}_{o}(t)>0,
\end{array}\right.
$$

where $\partial \Omega$ is the boundary of the set $\Omega$,

$$
\nabla f:=\frac{\partial f(\mathbf{r}, \hat{\mathbf{d}}(t))}{\partial \hat{\mathbf{d}}(t)}, \forall t
$$

and the convex function $f$ is defined in (14). This projection operator is used for constraining the estimated signal $\hat{\mathbf{d}}(t)$ into the convex set $\Omega$.

Using the DOB (17)-(19), the global-tracking control law handling the input constrains is proposed as

$$
\mathbf{u}(t)=\mathbf{K}_{s w} \hat{\mathbf{e}}(t)+\hat{\mathbf{u}}^{0}(\mathbf{r}, t), \forall t,
$$

where

$$
\begin{aligned}
\hat{\mathbf{e}}(t) & :=\mathbf{x}(t)-\hat{\mathbf{x}}^{0}(\mathbf{r}, t), \hat{\mathbf{x}}^{0}(\mathbf{r}, t):=\left.\mathbf{x}^{0}(\mathbf{r})\right|_{\mathbf{d}=\hat{\mathbf{d}}(t)}=\left[\begin{array}{c}
\hat{\mathbf{x}}_{1}^{0}(\mathbf{r}, t) \\
\mathbf{r}
\end{array}\right], \\
\mathbf{K}_{s w} & := \begin{cases}\mathbf{K}, & \text { if } \mathbf{K} \hat{\mathbf{e}}(t)+\hat{\mathbf{u}}^{0}(\mathbf{r}, t) \in U, \\
\mathbf{0}, & \text { otherwise, }\end{cases}
\end{aligned}
$$

$\mathbf{K}:=\mathbf{Y} \mathbf{Q}^{-1}$, and $\mathbf{Y}$ and $\mathbf{Q}$ are solutions of LMIs

$$
\begin{array}{r}
\mathbf{Q A}^{T}+\mathbf{Y}^{T} \mathbf{B}^{T}+\mathbf{A} \mathbf{Q}+\mathbf{B} \mathbf{Y}+\beta \mathbf{Q} \leq \mathbf{0} \\
\mathbf{Q A}^{T}+\mathbf{A Q}+\beta \mathbf{Q} \leq \mathbf{0}
\end{array}
$$

for some $\beta>0$ which is a design parameter. Figure 1 depicts the implemented closed-loop system.

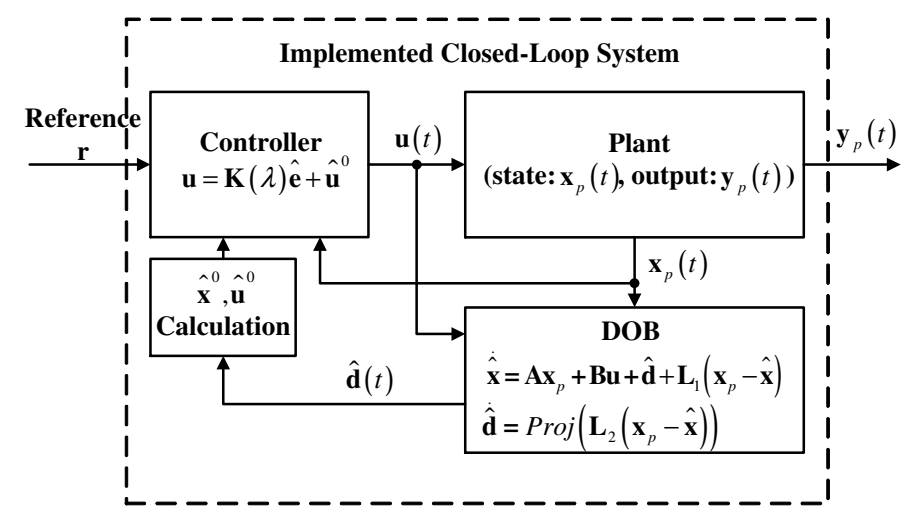

Figure 1. The implemented closed-loop system.

Note that the combination of DOB (17)-(19) and switching feedback gain $\mathbf{K}_{s w}$ critically plays the critical role in guaranteeing the global tracking and offset-free properties. See Section 4 for details.

\section{Stability Analysis}

This section asserts the global-tracking and offset-free properties from the proposed control law (20) including the DOB (17)-(19). Section 4.1 analyzes a DOB with a projection operator not only removing the steady state tracking error but also making the control input feasible for all time by using 
the projection operator. Section 4.2 proves the global-tracking and convergence properties. The proof of removal steady state errors is accomplished in Section 4.3.

\section{1. $D O B$}

The subtraction of (17) and (18) from (1) results in the error dynamics:

$$
\begin{aligned}
\dot{\mathbf{e}}_{o}(t) & =-\mathbf{L}_{1} \mathbf{e}_{o}(t)+\mathbf{e}_{d}(t), \\
\dot{\mathbf{e}}_{d}(t) & =-\operatorname{Proj}\left(\mathbf{L}_{2} \mathbf{e}_{o}(t)\right), \forall t,
\end{aligned}
$$

where $\mathbf{e}_{d}(t):=\mathbf{d}-\hat{\mathbf{d}}(t), \forall t$. Note that, because the estimation error dynamics (23)-(24) is not in the form of LTI system due to the projection operator in (24), it is not obvious to find the stabilizing DOB gains $\mathbf{L}_{1}$ and $\mathbf{L}_{2}$. Theorem 1 provides a guideline on how to choose stabilizing DOB gains for the estimation error dynamics (23)-(24).

Theorem 1. The estimation error dynamics comprised of (23)-(24) is globally asymptotically stable for any given $\mathbf{L}_{1}=\mathbf{L}_{1}^{T}>\mathbf{0}, \mathbf{L}_{2}=\mathbf{L}_{2}^{T}>\mathbf{0}$.

Proof. In order to analyze the stability of the estimation error dynamics, consider a scalar function given by

$$
\begin{aligned}
V_{o}\left(\mathbf{e}_{z}(t)\right) & =\frac{1}{2}\left\|\mathbf{e}_{o}(t)\right\|^{2}-l \mathbf{e}_{o}^{T}(t) \mathbf{e}_{d}(t)+\frac{1}{2} \mathbf{e}_{d}^{T}(t) \mathbf{L}_{2}^{-1} \mathbf{e}_{d}(t) \\
& :=\frac{1}{2} \mathbf{e}_{z}^{T}(t) \mathbf{P}_{o}(l) \mathbf{e}_{z}(t), \forall \mathbf{e}_{o}(0), \hat{\mathbf{d}}(0) \in \mathbb{R}^{n}
\end{aligned}
$$

where

$$
\begin{gathered}
l \in\left(0, \min \left\{\frac{1}{\sqrt{\lambda_{\max }\left(\mathbf{L}_{2}\right)}}, \frac{\lambda_{\min }\left(\mathbf{L}_{1}\right)}{\lambda_{\max }\left(\mathbf{L}_{2}+\frac{1}{4} \mathbf{L}_{1}^{2}\right)}\right\}\right), \\
\mathbf{e}_{z}(t):=\left[\begin{array}{c}
\mathbf{e}_{o}(t) \\
\mathbf{e}_{d}(t)
\end{array}\right], \mathbf{P}_{o}(l):=\left[\begin{array}{cc}
\mathbf{I}_{n \times n} & -l \mathbf{I}_{n \times n} \\
-l \mathbf{I}_{n \times n} & \mathbf{L}_{2}^{-1}
\end{array}\right] .
\end{gathered}
$$

$\mathbf{P}_{o}(l)$ is positive definite with $l$ in (26). Then, its time derivative along the trajectory of the state estimation error dynamics (23)-(24) becomes

$$
\begin{aligned}
\dot{V}_{o}\left(\mathbf{e}_{z}(t)\right)= & \mathbf{e}_{o}^{T}(t) \dot{\mathbf{e}}_{o}(t)-l \dot{\mathbf{e}}_{o}^{T}(t) \mathbf{e}_{d}(t)-l \mathbf{e}_{o}^{T}(t) \dot{\mathbf{e}}_{d}(t)+\mathbf{e}_{d}^{T}(t) \mathbf{L}_{2}^{-1} \dot{\mathbf{e}}_{d}(t) \\
= & \mathbf{e}_{o}^{T}(t)\left(-\mathbf{L}_{1} \mathbf{e}_{o}(t)+\mathbf{e}_{d}(t)\right)-l\left(-\mathbf{L}_{1} \mathbf{e}_{o}(t)+\mathbf{e}_{d}(t)\right)^{T} \mathbf{e}_{d}(t) \\
& +l \mathbf{e}_{o}^{T}(t) \operatorname{Proj}\left(\mathbf{L}_{2} \mathbf{e}_{o}(t)\right)-\mathbf{e}_{d}^{T}(t) \mathbf{L}_{2}^{-1} \operatorname{Proj}\left(\mathbf{L}_{2} \mathbf{e}_{o}(t)\right) .
\end{aligned}
$$

Note that it is easily verified that

$$
\begin{aligned}
\mathbf{e}_{o}^{T}(t) \operatorname{Proj}\left(\mathbf{L}_{2} \mathbf{e}_{o}(t)\right) & \leq \mathbf{e}_{o}^{T}(t) \mathbf{L}_{2} \mathbf{e}_{o}(t), \\
-\mathbf{e}_{d}^{T}(t) \mathbf{L}_{2}^{-1} \operatorname{Proj}\left(\mathbf{L}_{2} \mathbf{e}_{o}(t)\right) & \leq-\mathbf{e}_{d}^{T}(t) \mathbf{L}_{2}^{-1} \mathbf{L}_{2} \mathbf{e}_{o}(t), \forall t,
\end{aligned}
$$

using the definition of the projection operator (19). For details of the two properties (27)-(28), see Appendix E in Reference [21]. It follows from the two properties (27)-(28) that

$$
\begin{aligned}
\dot{V}_{o}\left(\mathbf{e}_{z}(t)\right) & \leq-\mathbf{e}_{o}^{T}(t)\left(\mathbf{L}_{1}-l \mathbf{L}_{2}\right) \mathbf{e}_{o}(t)+l \mathbf{e}_{o}^{T}(t) \mathbf{L}_{1} \mathbf{e}_{d}(t)-l\left\|\mathbf{e}_{d}(t)\right\|^{2} \\
& =:-\mathbf{e}_{z}^{T}(t) \mathbf{Q}_{o}(l) \mathbf{e}_{z}(t)
\end{aligned}
$$


where

$$
\mathbf{Q}_{o}(l):=\left[\begin{array}{cc}
\mathbf{L}_{1}-l \mathbf{L}_{2} & -\frac{l}{2} \mathbf{L}_{1} \\
-\frac{l}{2} \mathbf{L}_{1} & l \mathbf{I}_{n \times n}
\end{array}\right] .
$$

Note that $\mathbf{Q}_{o}(l)$ is also positive definite with $l$ in (26). Therefore, this means that the DOB (17)-(18) makes the estimation error dynamics (23)-(24) globally asymptotically stable with $l$ in (26). As a matter of fact, the interval in (26) is determined such that both $\mathbf{P}_{o}(l)$ and $\mathbf{Q}_{o}(l)$ become positive definite for any constant $l$ in the interval (this can be proved easily).

Remark 3. Although the DOB works with any positive definite $\mathbf{L}_{1}$ and $\mathbf{L}_{2}$, there can be many different ways to choose them. For instance, it is possible to find stabilizing DOB gains $\mathbf{L}_{1}$ and $\mathbf{L}_{2}$ for maximizing the decay rate of the estimation error. By letting the projection operator of (18) the identity function, the estimation error dynamics (23)-(24) can be written as

$$
\dot{\mathbf{e}}_{o b s}(t)=\left(\mathbf{A}_{o b s}-\mathbf{L}_{o b s} \mathbf{C}_{o b s}\right) \mathbf{e}_{o b s}(t), \forall t,
$$

where

$$
\mathbf{e}_{o b s}(t)=\left[\begin{array}{l}
\mathbf{e}_{o}(t) \\
\mathbf{e}_{d}(t)
\end{array}\right], \mathbf{A}_{o b s}=\left[\begin{array}{ll}
\mathbf{0}_{n \times n} & \mathbf{I}_{n \times n} \\
\mathbf{0}_{n \times n} & \mathbf{0}_{n \times n}
\end{array}\right], \mathbf{C}_{o b s}=\left[\begin{array}{ll}
\mathbf{I}_{n \times n} & \mathbf{0}_{n \times n}
\end{array}\right], \mathbf{L}_{o b s}=\left[\begin{array}{l}
\mathbf{L}_{1} \\
\mathbf{L}_{2}
\end{array}\right] .
$$

Then, the optimization problem given by

$$
\begin{gathered}
\max _{\alpha>0, \mathbf{P}_{o b s}=\mathbf{P}_{o b s}^{T}>\mathbf{0}, \mathbf{L}_{i}=\mathbf{L}_{i}^{T}>\mathbf{0}, i=1,2} \text { subject to } \\
\mathbf{A}_{o b s}^{T} \mathbf{P}_{o b s}-\mathbf{C}_{o b s}^{T} \mathbf{Y}_{o b s}^{T}+\mathbf{P}_{o b s} \mathbf{A}_{o b s}-\mathbf{Y}_{o b s} \mathbf{C}_{o b s}+\alpha \mathbf{P}_{o b s} \leq \mathbf{0}
\end{gathered}
$$

yields the optimal DOB gain $\mathbf{L}_{o b s}^{*}=\left(\mathbf{P}_{o b s}^{*}\right)^{-1} \mathbf{Y}_{o b s}^{*}$ maximizing the decay rate of the estimation error $\mathbf{e}_{o b s}(t)$ of (30) where $\left(\alpha^{*}, \mathbf{P}_{o b s}^{*}, \mathbf{Y}_{o b s}^{*}\right)$ represents the optimizer of the optimization problem (31).

\subsection{Input Constrained Offset-Free Global Tracking Controller}

The estimated steady state and control (16) play the pivotal role in proving both the closed-loop stability and feasibility for all time. On the other hand, the estimated nominal steady state input $\hat{\mathbf{u}}^{0}(\mathbf{r}, t)$ can be interpreted as an estimated steady state input based on the estimated signal $\hat{\mathbf{d}}(t)$. Hence, it is important for $\hat{\mathbf{u}}^{0}(\mathbf{r}, t)$ to be feasible for admissibility. In fact, the projection operator in the DOB makes $\hat{\mathbf{u}}^{0}(\mathbf{r}, t)$ feasible for all time. Moreover, the projection operator in the DOB guarantees that

$$
\forall \hat{\mathbf{d}}(0) \in \Omega^{o} \stackrel{\text { implies }}{\longrightarrow} \hat{\mathbf{d}}(t) \in \Omega, \forall t
$$

owing to the projection operator [21]. Due to this invariance property, $\hat{\mathbf{u}}^{0}(\mathbf{r}, t)$ becomes feasible for all time as shown in Lemma 1.

Lemma 1. For an admissible reference $r$, assuming that the initial condition $\hat{\boldsymbol{d}}(0)$ is constrained into $\Omega^{o}$, it holds that

$$
\hat{\boldsymbol{u}}^{0}(\boldsymbol{r}, t) \in U, \forall t .
$$


Proof. Using the property (33), the following chain implications hold:

$$
\begin{aligned}
\forall \hat{\mathbf{d}}(0) \in \Omega^{o} \Rightarrow \hat{\mathbf{d}}(t) \in \Omega, \forall t . & \Leftrightarrow f(\mathbf{r}, \hat{\mathbf{d}}(t)) \leq 0, \forall t . \\
& \Leftrightarrow\left\|\mathbf{D r}+\mathbf{Z}_{2} \hat{\mathbf{d}}(t)-\mathbf{u}_{c}\right\| \leq r_{u}, \forall t . \\
& \Leftrightarrow\left\|\hat{\mathbf{u}}^{0}(\mathbf{r}, t)-\mathbf{u}_{c}\right\| \leq r_{u}, \forall t . \\
& \Leftrightarrow \hat{\mathbf{u}}^{0}(\mathbf{r}, t) \in U_{c} \subseteq U, \forall t . \\
& \Rightarrow \hat{\mathbf{u}}^{0}(\mathbf{r}, t) \in U, \forall t,
\end{aligned}
$$

where the third implication comes from (15).

Now it is ready to show the global convergence of the closed-loop error dynamics.

Theorem 2. For an admissible reference $\mathbf{r}$ and any given $\mathbf{L}_{1}=\mathbf{L}_{1}^{T}>\mathbf{0}, \mathbf{L}_{2}=\mathbf{L}_{2}^{T}>\mathbf{0}, \hat{\mathbf{d}}(0) \in \Omega^{o}$, and $\mathbf{x}(0) \in \mathbb{R}^{n}$, suppose that the two LMIs (21)-(22) are feasible. Then, the proposed controller (20) guarantees to achieve the constrained global asymptotic output tracking property described in Definition 1.

Proof. Since $\hat{\mathbf{d}}(0) \in \Omega^{o}$, Lemma 1 holds true. Therefore, the control input is always feasible (C3 of Definition 1). In addition, the proposed controller (20) can be written by applying the two equalities (16) as

$$
\begin{aligned}
\mathbf{u}(t) & =\mathbf{K}_{s w} \hat{\mathbf{e}}(t)+\hat{\mathbf{u}}^{0}(\mathbf{r}, t) \\
& =\mathbf{K}_{s w} \mathbf{e}(t)+\mathbf{u}^{0}(\mathbf{r}, \mathbf{d})+\mathbf{K}_{s w}\left(\mathbf{x}^{0}(\mathbf{r}, \mathbf{d})-\hat{\mathbf{x}}^{0}(\mathbf{r}, t)\right)-\left(\mathbf{u}^{0}(\mathbf{r}, \mathbf{d})-\hat{\mathbf{u}}^{0}(\mathbf{r}, t)\right) \\
& =: \mathbf{K}_{s w} \mathbf{e}(t)+\mathbf{u}^{0}(\mathbf{r}, \mathbf{d})+\mathbf{T}(t) \mathbf{e}_{d}(t), \forall t,
\end{aligned}
$$

where

$$
\mathbf{e}(t):=\mathbf{x}(t)-\mathbf{x}^{0}(\mathbf{r}, \mathbf{d}), \mathbf{T}(t):=\mathbf{K}_{s w}\left[\begin{array}{cc}
\mathbf{Z}_{11} & \mathbf{Z}_{12} \\
\mathbf{0}_{m \times(n-m)} & \mathbf{0}_{m \times m}
\end{array}\right]-\left[\begin{array}{ll}
\mathbf{Z}_{21} & \mathbf{Z}_{22}
\end{array}\right], \forall t .
$$

Note that, since the control gain $\mathbf{K}_{s w}$ is a kind of a time-varying signal, the matrix $\mathbf{T}(t)$ is also defined as a time-varying signal. Then, the corresponding closed-loop error dynamics is obtained as

$$
\dot{\mathbf{e}}(t)=\left(\mathbf{A}+\mathbf{B} \mathbf{K}_{s w}\right) \mathbf{e}(t)+\mathbf{B T}(t) \mathbf{e}_{d}(t), \forall t,
$$

where $\mathbf{e}_{d}(t)$ satisfies the differential equation described in (18). This error dynamics is a switched linear system owing to the switching gain $\mathbf{K}_{s w}$. Therefore, in order to analyze the switched system, consider the positive definite function as a candidate for the common Lyapunov function in the following:

$$
\begin{aligned}
V\left(\mathbf{e}(t), \mathbf{e}_{z}(t)\right) & :=\frac{1}{2} \mathbf{e}^{T}(t) \mathbf{P e}(t)+k V_{o}\left(\mathbf{e}_{z}(t)\right) \\
& =\frac{1}{2} \mathbf{e}^{T}(t) \mathbf{P e}(t)+\frac{k}{2} \mathbf{e}_{z}^{T}(t) \mathbf{P}_{o}(l) \mathbf{e}_{z}(t),
\end{aligned}
$$

where $\mathbf{P}:=\mathbf{Q}^{-1}$, the positive constant $k$ will be defined later in order to ensure $V\left(\mathbf{e}(t), \mathbf{e}_{z}(t)\right)$ to be monotonically decreasing for all time, and the parameter $l$ is chosen to be in the interval of (26) so that the two matrices $\mathbf{P}_{o}(l)$ and $\mathbf{Q}_{o}(l)$ are both positive definite for given $\mathbf{L}_{1}=\mathbf{L}_{1}^{T}>\mathbf{0}$ and $\mathbf{L}_{2}=\mathbf{L}_{2}^{T}>\mathbf{0}$. It should be noticed that the initial condition $\hat{\mathbf{d}}(0)$ is constrained into the set $\Omega^{o}$ for guaranteeing the 
feasibility of the proposed control input (see Lemma 1). Then, it follows from the Equation (34) and the inequality (29) of Theorem 1 that

$$
\begin{aligned}
\dot{V} \leq & \frac{1}{2} \mathbf{e}^{T}(t)\left(\left(\mathbf{A}+\mathbf{B} \mathbf{K}_{s w}\right)^{T} \mathbf{P}+\mathbf{P}\left(\mathbf{A}+\mathbf{B} \mathbf{K}_{s w}\right)\right) \mathbf{e}(t)+\mathbf{e}^{T}(t) \mathbf{T}^{T}(t) \mathbf{B}^{T} \mathbf{P B T}(t) \mathbf{e}_{d}(t) \\
& -k \mathbf{e}_{z}^{T}(t) \mathbf{Q}_{o}(l) \mathbf{e}_{z}(t) .
\end{aligned}
$$

Note that the first linear matrix inequality (LMI) (21) leads to

$$
\left[\left(\mathbf{A}+\mathbf{B K}_{s w}\right)^{T} \mathbf{P}+\mathbf{P}\left(\mathbf{A}+\mathbf{B} K_{s w}\right)\right]_{\mathbf{K}_{s w}=\mathbf{K}=\mathbf{Y Q}^{-1}}=(\mathbf{A}+\mathbf{B K})^{T} \mathbf{P}+\mathbf{P}(\mathbf{A}+\mathbf{B K}) \leq-\beta \mathbf{P}
$$

and the second LMI (22) does

$$
\left[\left(\mathbf{A}+\mathbf{B} \mathbf{K}_{s w}\right)^{T} \mathbf{P}+\mathbf{P}\left(\mathbf{A}+\mathbf{B} \mathbf{K}_{s w}\right)\right]_{\mathbf{K}_{s w}=\mathbf{0}}=\mathbf{A}^{T} \mathbf{P}+\mathbf{P A} \leq-\beta \mathbf{P} .
$$

Hence, $\dot{V}$ satisfies that

$$
\begin{aligned}
\dot{V} & \leq-\frac{\beta}{2} \mathbf{e}^{T}(t) \mathbf{P e}(t)+\mathbf{e}^{T}(t) \mathbf{T}^{T}(t) \mathbf{B}^{T} \mathbf{P B T}(t) \mathbf{e}_{d}(t)-k \mathbf{e}_{z}^{T}(t) \mathbf{Q}_{o}(l) \mathbf{e}_{z}(t) \\
& \leq-\frac{\beta}{4} \mathbf{e}^{T}(t) \mathbf{P e}(t)+\frac{\left\|\mathbf{T}(t)^{T} \mathbf{B}^{T} \mathbf{P B T}(t)\right\|^{2}}{\beta \lambda_{\min }(\mathbf{P})}\left\|\mathbf{e}_{z}(t)\right\|^{2}-k \mathbf{e}_{z}^{T}(t) \mathbf{Q}_{o}(l) \mathbf{e}_{z}(t)
\end{aligned}
$$

where the second inequality comes from

$$
\left\|\mathbf{e}_{d}(t)\right\|^{2} \leq\left\|\mathbf{e}_{z}(t)\right\|^{2}, \forall t,
$$

and the Young's inequality [22], i.e.,

$$
\begin{aligned}
\mathbf{e}^{T}(t) \mathbf{T}(t){ }^{T} \mathbf{B}^{T} \mathbf{P B T}(t) \mathbf{e}_{d}(t) & \leq \frac{\lambda_{\min }(\mathbf{P}) \beta}{4}\|\mathbf{e}(t)\|^{2}+\frac{\left\|\mathbf{T}(t)^{T} \mathbf{B}^{T} \mathbf{P B T}(t)\right\|^{2}}{\lambda_{\min }(\mathbf{P}) \beta}\left\|\mathbf{e}_{d}(t)\right\|^{2} \\
& \leq \frac{\beta}{4} \mathbf{e}^{T}(t) \mathbf{P e}(t)+\frac{\left\|\mathbf{T}(t)^{T} \mathbf{B}^{T} \mathbf{P B T}(t)\right\|^{2}}{\lambda_{\min }(\mathbf{P}) \beta}\left\|\mathbf{e}_{z}(t)\right\|^{2}, \forall t
\end{aligned}
$$

$\lambda_{\min }((\cdot))$ and $\lambda_{\max }((\cdot))$ denote the minimum and maximum eigenvalues of the given square matrix $(\cdot)$, respectively. Defining the positive constant $k$ as

$$
k:=\left(\eta+\frac{\max _{t}\left\|\mathbf{T}(t)^{T} \mathbf{B}^{T} \mathbf{P B T}(t)\right\|^{2}}{\beta \lambda_{\min }(\mathbf{P}) \lambda_{\min }\left(\mathbf{Q}_{o}(l)\right)}\right), \forall \eta>0,
$$

we have

$$
\dot{V} \leq-\frac{\beta}{2} \mathbf{e}^{T}(t) \operatorname{Pe}(t)-\eta \mathbf{e}_{z}^{T}(t) \mathbf{Q}_{o}(l) \mathbf{e}_{z}(t)<0, \forall \mathbf{x}(0), \mathbf{e}_{o}(0) \in \mathbb{R}^{n}, \forall \hat{\mathbf{d}}(0) \in \Omega^{o}, \forall t,
$$

which means that $V$ is indeed a common Lyuapuov function for the switched system and that

$$
\lim _{t \rightarrow \infty} \mathbf{x}(t)=\mathbf{x}^{0}(\mathbf{r}, \mathbf{d}), \forall \mathbf{x}(0) \in \mathbb{R}^{n}(\mathrm{C} 2 \text { of Definition } 1) .
$$

It consequently results in

$$
\lim _{t \rightarrow \infty} \mathbf{y}(t)=\mathbf{r}, \forall \mathbf{x}(0) \in \mathbb{R}^{n}(\mathrm{C} 1 \text { of Definition } 1)
$$


Therefore, the proposed control law (20) ensures the constrained global asymptotic output tracking property.

Remark 4. The assumptions on the two matrices $\mathbf{A}$ and $\mathbf{B}$ are necessary for the proposed method to be applicable since the controllability of the pair $(\mathbf{A}, \mathbf{B})$ and the stability of the matrix $\mathbf{A}$ ensure the solvability of the two inequalities (21) and (22), respectively.

Remark 5. It is easy to see that the nominal proposed controller (20), $\left.\mathbf{u}(t)\right|_{\hat{\mathbf{d}}(k)=\mathbf{d}}$, gives the closed-loop error dynamics given by

$$
\dot{\mathbf{e}}(t)=\left(\mathbf{A}+\mathbf{B K}_{s w}\right) \mathbf{e}(t), \forall t .
$$

In this case, the two inequalities (21)-(22) renders the positive definite function

$$
V(\mathbf{e}(t))=\frac{1}{2} \mathbf{e}^{T}(t) \mathbf{P e}(t), \forall t,
$$

to satisfy

$$
\dot{V}(\mathbf{e}(t)) \leq-\frac{\beta}{2} V(\mathbf{e}(t)), \forall t,
$$

where $\mathbf{P}=\mathbf{Q}^{-1}$. Therefore, it is desirable to choose the two matrices $\mathbf{Q}$ and $\mathbf{Y}$ by solving the optimization problem

$$
\max _{\beta>0, \mathbf{Q}=\mathbf{Q}^{T}>\mathbf{0}, \mathbf{Y}} \text { subject to (21), (22), }
$$

for maximizing the decay rate of the tracking error.

Theorem 2 asserts that the proposed controller achieves the constrained global asymptotic output tracking property if there is no plant-model mismatch. In the next section, it is shown that the proposed controller actually results in offset-free tracking, as well, even for the case where there is plant-model mismatch.

\subsection{Offset Error Rejection for Plant-Model Mismatched Case}

Theorem 3 claims that the proposed controller (20) guarantees the offset-free property as long as the implemented closed-loop system eventually reaches a steady state as the time goes to infinity, i.e.,

$$
\begin{aligned}
\lim _{t \rightarrow \infty} \mathbf{x}_{p}(t) & =\mathbf{x}_{p, \infty}\left(=\left[\begin{array}{c}
\mathbf{x}_{p 1, \infty} \\
\mathbf{y}_{p, \infty}
\end{array}\right]\right), \lim _{t \rightarrow \infty} \hat{\mathbf{x}}(t)=\hat{\mathbf{x}}_{\infty}, \\
\lim _{t \rightarrow \infty} \mathbf{u}(t) & =\mathbf{u}_{\infty}, \lim _{t \rightarrow \infty} \hat{\mathbf{d}}(t)=\hat{\mathbf{d}}_{\infty} \in \Omega^{o},
\end{aligned}
$$

where $\mathbf{x}_{p}(t)$ represents the state trajectory of the implemented closed-loop system in Figure 1.

Theorem 3. Suppose that the all assumptions of Theorem 2 hold and that the implemented closed-loop system shown in Figure 1 satisfies the condition (40) in the steady state. Then, it holds that

$$
\mathbf{y}_{p, \infty}=\mathbf{r}, \forall \mathbf{x}(0) \in \mathbb{R}^{n}
$$

if matrix $\mathbf{W}$ is invertible where the matrix $\mathbf{W}$ is defined as

$$
W:=\mathbf{K}_{\infty}\left[\begin{array}{c}
\mathbf{Z}_{11} \mathbf{A}_{12}+\mathbf{Z}_{12} \mathbf{A}_{22} \\
\mathbf{I}_{m \times m}
\end{array}\right]-\mathbf{Z}_{21} \mathbf{A}_{12}-\mathbf{Z}_{22} \mathbf{A}_{22}
$$

and $\mathbf{K}_{\infty}$ is the steady state value of $\mathbf{K}_{\text {sw }}$, i.e., either $\mathbf{0}$ or $\mathbf{Y} \mathbf{Q}^{-1}$. 
Proof. In the steady state described as (40), the DOB (17)-(18) and the Equation (15) can be written as

$$
\begin{aligned}
\mathbf{0} & =\mathbf{A} \mathbf{x}_{p, \infty}+\mathbf{B} \mathbf{u}_{\infty}+\hat{\mathbf{d}}_{\infty}+\mathbf{L}_{1}\left(\mathbf{x}_{p, \infty}-\hat{\mathbf{x}}_{\infty}\right) \\
\mathbf{0} & =\operatorname{Proj}\left(\mathbf{L}_{2}\left(\mathbf{x}_{p, \infty}-\hat{\mathbf{x}}_{\infty}\right)\right) \\
{\left[\begin{array}{c}
\hat{\mathbf{x}}_{1, \infty}^{0} \\
\hat{\mathbf{u}}_{\infty}^{0}
\end{array}\right] } & =\left[\begin{array}{ll}
\mathbf{Z}_{11} & \mathbf{Z}_{12} \\
\mathbf{Z}_{21} & \mathbf{Z}_{22}
\end{array}\right]\left[\begin{array}{c}
\mathbf{A}_{12} \\
\mathbf{A}_{22}
\end{array}\right] \mathbf{r}+\left[\begin{array}{ll}
\mathbf{Z}_{11} & \mathbf{Z}_{12} \\
\mathbf{Z}_{21} & \mathbf{Z}_{22}
\end{array}\right] \hat{\mathbf{d}}_{\infty} .
\end{aligned}
$$

Since $\hat{\mathbf{d}}_{\infty} \in \Omega^{o}$, it follows from the Equation (43) that

$$
\mathbf{L}_{2}\left(\mathbf{x}_{p, \infty}-\hat{\mathbf{x}}_{\infty}\right)=\mathbf{0}
$$

It implies that

$$
\mathbf{x}_{p, \infty}=\hat{\mathbf{x}}_{\infty}
$$

since the matrix $\mathbf{L}_{2}$ is invertible. It is easy to see that the property (45) makes it possible for the Equation (42) to be written as

$$
\mathbf{0}=\mathbf{A} \mathbf{x}_{p, \infty}+\mathbf{B} \mathbf{u}_{\infty}+\hat{\mathbf{d}}_{\infty} .
$$

Since this equation can be equivalently expressed as

$$
\left[\begin{array}{c}
\mathbf{x}_{p 1, \infty} \\
\mathbf{u}_{\infty}
\end{array}\right]=\left[\begin{array}{ll}
\mathbf{Z}_{11} & \mathbf{Z}_{12} \\
\mathbf{Z}_{21} & \mathbf{Z}_{22}
\end{array}\right]\left[\begin{array}{l}
\mathbf{A}_{12} \\
\mathbf{A}_{22}
\end{array}\right] \mathbf{y}_{\infty}+\left[\begin{array}{ll}
\mathbf{Z}_{11} & \mathbf{Z}_{12} \\
\mathbf{Z}_{21} & \mathbf{Z}_{22}
\end{array}\right] \hat{\mathbf{d}}_{\infty}
$$

subtracting (44) from (46), we have

$$
\begin{aligned}
\mathbf{x}_{p 1, \infty}-\hat{\mathbf{x}}_{1, \infty}^{0} & =\left(\mathbf{Z}_{11} \mathbf{A}_{12}+\mathbf{Z}_{12} \mathbf{A}_{22}\right)\left(\mathbf{y}_{\infty}-\mathbf{r}\right), \\
\mathbf{u}_{\infty}-\hat{\mathbf{u}}_{\infty}^{0} & =\left(\mathbf{Z}_{21} \mathbf{A}_{12}+\mathbf{Z}_{22} \mathbf{A}_{22}\right)\left(\mathbf{y}_{\infty}-\mathbf{r}\right) .
\end{aligned}
$$

Now consider the control input in the steady state as follows:

$$
\begin{aligned}
\mathbf{u}_{\infty} & =\lim _{k \rightarrow \infty}\left(\mathbf{K}_{s w} \hat{\mathbf{e}}(t)+\hat{\mathbf{u}}^{0}(\mathbf{r}, t)\right) \\
& =\mathbf{K}_{\infty}\left(\mathbf{x}_{p, \infty}-\hat{\mathbf{x}}_{\infty}^{0}\right)+\hat{\mathbf{u}}_{\infty}^{0} \\
& =\mathbf{K}_{\infty}\left[\begin{array}{c}
\mathbf{x}_{p 1, \infty}-\hat{\mathbf{x}}_{1, \infty}^{0} \\
\mathbf{y}_{\infty}-\mathbf{r}
\end{array}\right]-\left(\mathbf{u}_{\infty}-\hat{\mathbf{u}}_{\infty}^{0}\right)+\mathbf{u}_{\infty} .
\end{aligned}
$$

It implies, together with the Equations (47), that

$$
\begin{aligned}
\mathbf{u}_{\infty}-\hat{\mathbf{u}}_{\infty}^{0} & =\mathbf{K}_{\infty}\left[\begin{array}{c}
\mathbf{x}_{p 1, \infty}-\hat{\mathbf{x}}_{1, \infty}^{0} \\
\mathbf{y}_{\infty}-\mathbf{r}
\end{array}\right] \\
& =\mathbf{K}_{\infty}\left[\begin{array}{c}
\mathbf{Z}_{11} \mathbf{A}_{12}+\mathbf{Z}_{12} \mathbf{A}_{22} \\
\mathbf{I}_{m \times m}
\end{array}\right]\left(\mathbf{y}_{\infty}-\mathbf{r}\right) .
\end{aligned}
$$

It follows from equating (48) and (49) that

$$
\begin{aligned}
\left(\mathbf{K}_{\infty}\left[\begin{array}{c}
\mathbf{Z}_{11} \mathbf{A}_{12}+\mathbf{Z}_{12} \mathbf{A}_{22} \\
\mathbf{I}_{m \times m}
\end{array}\right]-\mathbf{Z}_{21} \mathbf{A}_{12}-\mathbf{Z}_{22} \mathbf{A}_{22}\right)\left(\mathbf{y}_{\infty}-\mathbf{r}\right) & =\mathbf{W}\left(\mathbf{y}_{\infty}-\mathbf{r}\right) \\
& =\mathbf{0} .
\end{aligned}
$$

Therefore, the invertibility of the matrix $\mathbf{W}$ guarantees that $\mathbf{y}_{\infty}=\mathbf{r}$. 
In this theorem, it is shown that the steady state offset is indeed removed by the proposed DOB based control even for uncertain systems as long as the uncertain system reaches at a steady state.

\section{An Application for a DC/DC Buck Converter}

Using the proposed method, this section designs a state-feedback controller for a DC/DC buck converter to control the output voltage under the inherent input constraints and performs the simulations to show the effectiveness of the proposed method using the PowerSIM(PSIM) software (9.1, Powersim Inc, Rockville, MD, USA).

\subsection{Description of a DC/DC Converter}

Figure 2 describes the topology of the DC/DC buck converter which is utilized for lowering the source DC voltage to the desired DC voltage by controlling the switch device $Q_{1}[23,24]$.

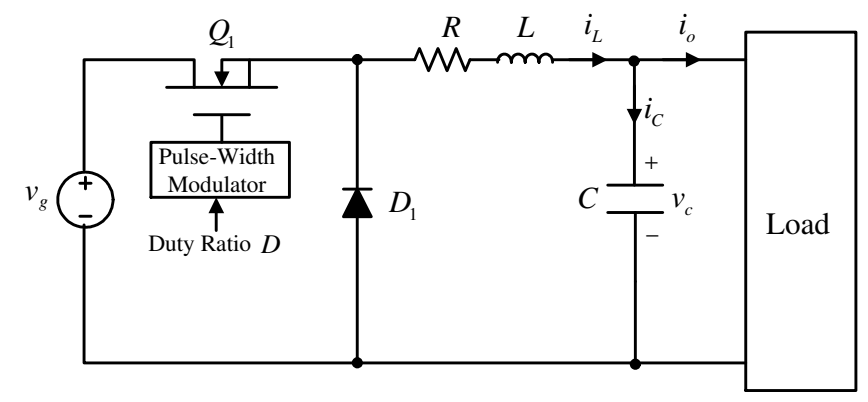

Figure 2. The DC/DC buck converter topology.

In this system, the pulse width modulation (PWM) of the switching action is considered as the control input, i.e., if the duty ratio is $D \in[0,1]$, the switch turns on for a period of $D T$ and turns off for a period of $(1-D) T$, where $T$ is the PWM period. Therefore, the averaged model of this buck converter is obtained as follows:

$$
\dot{\mathbf{x}}(t)=\mathbf{A x}(t)+\mathbf{B} u(t)+\mathbf{d}(t)
$$

where $u(t):=D \in[0,1]$,

$$
\begin{aligned}
\mathbf{x}(t) & :=\left[\begin{array}{c}
i_{L}(t) \\
v_{C}(t)
\end{array}\right], \mathbf{v}:=\left[\begin{array}{c}
v_{g} \\
v_{D}
\end{array}\right], \mathbf{d}(t):=\mathbf{B}_{v} \mathbf{v}+\mathbf{B}_{d} i_{o}(t), \\
\mathbf{A} & :=\left[\begin{array}{cc}
-\frac{R}{L} & -\frac{1}{L} \\
\frac{1}{C} & 0
\end{array}\right], \mathbf{B}:=\left[\begin{array}{c}
\frac{1}{L}\left(v_{g}+v_{D}\right) \\
0
\end{array}\right], \\
\mathbf{B}_{v} & :=\left[\begin{array}{cc}
0 & -\frac{1}{L} \\
0 & 0
\end{array}\right], \mathbf{B}_{d}:=\left[\begin{array}{c}
0 \\
-\frac{1}{C}
\end{array}\right],
\end{aligned}
$$

$v_{D}$ represents the diode voltage.

In order to control the output voltage $\mathbf{v}_{o}(t)$, define the output variable

$$
\mathbf{y}(t):=\mathbf{C x}(t),
$$

where $\mathbf{C}:=\left[\begin{array}{ll}0 & 1\end{array}\right]$. Because, in general, since it is possible to assume that the load current $i_{o}(t)$ is unknown but constant [25], the vector $\mathbf{d}(t)$ can be considered as the unknown constant $\mathbf{d}$ as in (1).

Note that, since the open-loop system matrix $\mathbf{A}$ is stable for any $R>0, L>0, C>0$, and that the pair $(\mathbf{A}, \mathbf{B})$ is controllable, the necessary condition for finding the solution of the optimization problem (39) is satisfied. The minimum and maximum duty ratio 0 and 1 correspond to the lower 
and upper input constraint $\underline{\mathbf{u}}$ and $\overline{\mathbf{u}}$, respectively, and the control objective for the buck converter is to design the control input $u(t)$ so that

$$
\lim _{t \rightarrow \infty} y(t)=r
$$

for a given output voltage reference $r$ under the input constraints (5).

\subsection{Simulations}

The DC/DC buck converter parameters are given by

$$
R=0.1 \Omega, L=3 m H, C=2000 \mu F, v_{g}=250 \mathrm{~V}, v_{D}=0.67 \mathrm{~V} .
$$

It was checked that the optimization problem (39) yields the optimal solution $\beta^{*}=33$ with

$$
\mathbf{Q}^{*}=10^{3}\left[\begin{array}{cc}
6.65 & -0.33 \\
-0.33 & 9.96
\end{array}\right] \text { and } \mathbf{Y}^{*}=\left[\begin{array}{ll}
-63.74 & -0.033
\end{array}\right] .
$$

The estimation gain matrices of the DOB are obtained

$$
\mathbf{L}_{1}=3.2 \times 10^{3} \mathbf{I}_{2 \times 2}, \mathbf{L}_{2}=6.3 \times 10^{5} \mathbf{I}_{2 \times 2}
$$

by solving the optimization problem (31). In this application, bearing the input constraint $u(t) \in[0,1]$ in mind, the convex function $f$ and the convex set $\Omega$ for the projection operator are constructed as

$$
\begin{aligned}
f(r, \mathbf{d}) & =\left\|\mathbf{D} r+\mathbf{Z}_{2} \mathbf{d}-0.5\right\|^{2}-0.5^{2}, \\
\Omega & =\{\mathbf{d} \mid f(r, \mathbf{d}) \leq 0\} .
\end{aligned}
$$

The corresponding $\nabla f, \Omega^{\circ}$, and $\partial \Omega$ are given by

$$
\nabla f=2 \mathbf{Z}_{2}^{T}\left(\mathbf{D} r+\mathbf{Z}_{2} \mathbf{d}-0.5\right), \Omega^{o}=\{\mathbf{d} \mid f(r, \mathbf{d})<0\}, \partial \Omega:=\{\mathbf{d} \mid f(r, \mathbf{d})=0\} .
$$

Together with these settings, the projection operator was implemented according to its definition (19), and the proposed controller (20) with the DOB (17)-(18) were implemented by using the DLL block in the PSIM software with the sampling time of $0.1 \mathrm{~ms}$, and the PWM switching frequency was chosen as $10 \mathrm{kHz}$. For zero steady state errors as stated in Theorem 3, we also checked the matrix $\mathbf{W}$ defined in (41) is invertible using the parameters used in this simulation.

In order to compare the closed-loop performance, we consider the saturated global stabilizing integral controller in Reference [15], given by

$$
u_{\text {int }}(t)=-s a t\left(k \mathbf{B}_{\text {int }}^{T} \mathbf{P}_{\text {int }} \mathbf{z}_{\text {int }}(t)\right), k>0, \forall t
$$

with the tuned design parameter $k=10$ for the best convergence rate with $\pm 10 \%$ overshoot through ad-hoc method, where the saturation function sat $(\mathbf{x})$ is defined as

$$
\operatorname{sat}(\mathbf{x})=\left[\begin{array}{lll}
\operatorname{sat}\left(x_{1}\right) & \cdots & \operatorname{sat}\left(x_{n}\right)
\end{array}\right]^{T}
$$

with

$$
\operatorname{sat}\left(x_{i}\right)=\left\{\begin{array}{l}
u_{\max }, x_{i}>u_{\max } \\
x_{i}, u_{\min } \leq x_{i} \leq u_{\max } \\
u_{\min }, x_{i}<u_{\min }
\end{array}\right.
$$


for any given $\mathbf{x} \in \mathbb{R}^{n}, \mathbf{B}_{\text {int }}=\left[\begin{array}{ll}\mathbf{B}^{T} & 0\end{array}\right]^{T}$, the matrix $\mathbf{P}_{\text {int }}$ is chosen to be

$$
\mathbf{P}_{\text {int }}=10^{-4}\left[\begin{array}{ccc}
0.2 & 0 & -1.2 \\
0 & 0.1 & -0.1 \\
-1.2 & -0.1 & 387.5
\end{array}\right] \quad\left(=\mathbf{P}_{\text {int }}^{T}>\mathbf{0}\right)
$$

so that it satisfies the inequality

$$
\mathbf{A}_{\text {int }}^{T} \mathbf{P}_{\text {int }}+\mathbf{P}_{\text {int }} \mathbf{A}_{\text {int }} \leq \mathbf{0}
$$

with $\mathbf{A}_{\text {int }}=\left[\begin{array}{cc}\mathbf{A} & \mathbf{0}_{2 \times 1} \\ -\mathbf{C} & 0\end{array}\right], \mathbf{z}_{\text {int }}(t)=\left[\begin{array}{ll}\mathbf{x}^{T}(t) & s(t)\end{array}\right]^{T}$, and $s(t)$ is the integral state governed by the dynamics:

$$
\dot{s}(t)=r-y(t), \forall t
$$

Furthermore, for more realistic simulations, we used the perturbed parameters $R_{p}, L_{p}$, and $C_{p}$ given by

$$
R_{p}=0.05 \Omega(=0.5 R), L_{p}=4 m H(=1.3 L), C_{p}=1700 \mu F(=0.85 C),
$$

instead of their true parameters $R, L$, and $C$, respectively.

Under these settings, the simulation scenario is given by

1. The control commences with the output voltage reference $r=230 \mathrm{~V}$ and with the resistive load $R_{L}=10 \Omega$ at $t=0 \mathrm{~s}$,

2. The output voltage reference $r$ is decreased from $r=230 \mathrm{~V}$ to $r=30 \mathrm{~V}$ at $t=0.2 \mathrm{~s}$,

3. The output voltage reference $r$ is increased from $r=30 \mathrm{~V}$ to $r=230 \mathrm{~V}$ at $t=0.4 \mathrm{~s}$,

4. The resistive load is decreased from $R_{L}=10 \Omega$ to $R_{L}=5 \Omega$ at $t=0.6 \mathrm{~s}$, and

5. The resistive load value is decreased from $R_{L}=5 \Omega$ to $R_{L}=2.5 \Omega$ at $t=0.8 \mathrm{~s}$.

Figure 3 compare the output voltage tracking performances between the proposed controller and the integral controller. This result shows that the proposed method provides an effective output tracking performance without any offset error in the presence of a plant-model mismatch as comparing the integral controller. In particular, it was observed that the proposed method keeps the output voltage tracking performance satisfactory as comparing the integral control method, despite the severe load variations.

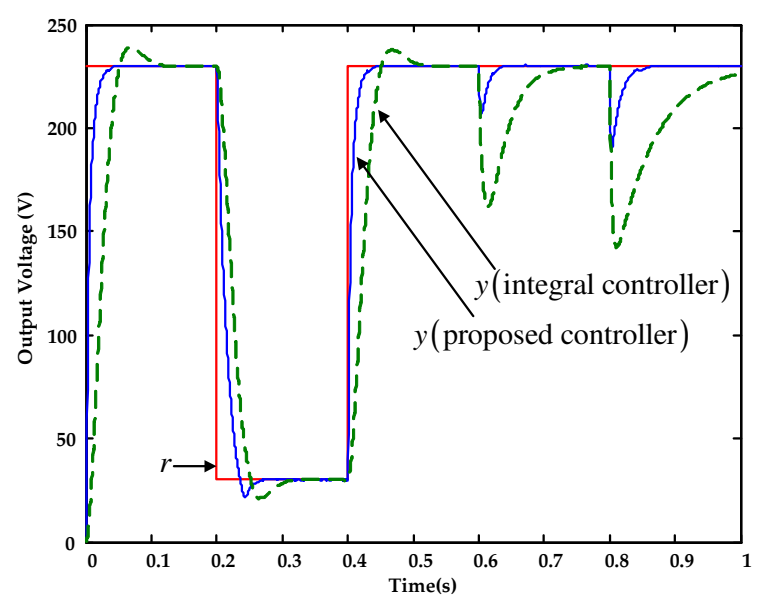

Figure 3. The output voltage tracking performance comparison. 
Figure 4 shows the corresponding control input behavior and the state estimation errors. The control input satisfies the input constraints for all time and that the DOB effectively estimates the real states in the realistic simulations, thanks to the integrator in (18), respectively.
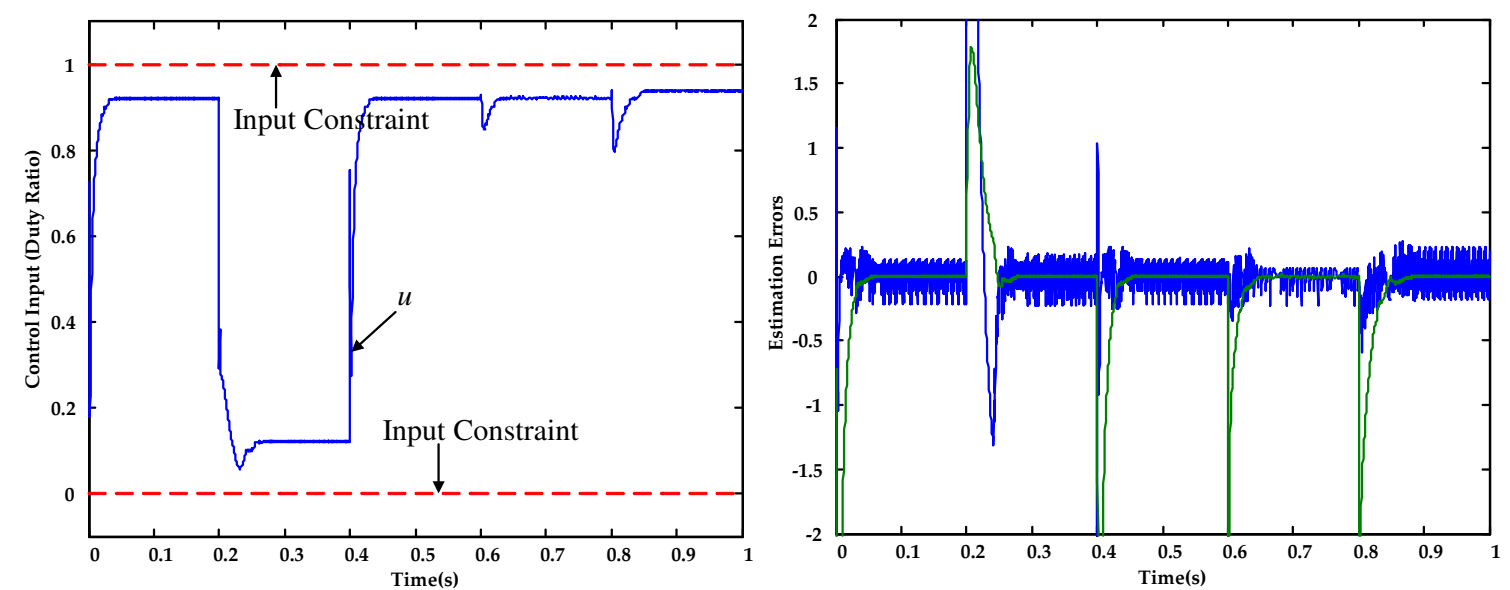

Figure 4. The behaviors of control input and state estimation.

Under the same simulation scenario used in the first one, the second simulation was performed to show the saturation effect of the control input. To this end, the matrices $\mathbf{Q}$ and $\mathbf{Y}$ for the control gain were selected as

$$
\mathbf{Q}=10^{-3}\left[\begin{array}{cc}
0.1 & -0.005 \\
-0.005 & 0.15
\end{array}\right] \text { and } \mathbf{Y}=\left[\begin{array}{ll}
-9 & 0
\end{array}\right]
$$

to satisfy the inequalities in (22) so that the resulting control gain makes the closed-loop system oscillatory. The output voltage response is shown in Figure 5, respectively.

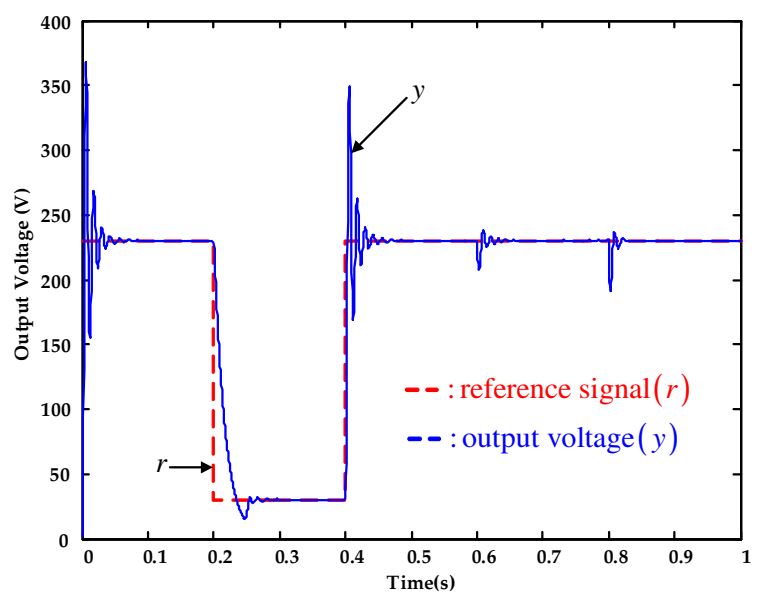

Figure 5. The output tracking performance.

Figure 6 shows that the corresponding control input still satisfies the input constraint for all time where the right panel magnifies the control input behavior in the left panel during the starting transient period $0 \sim 0.3 \mathrm{~s}$. 

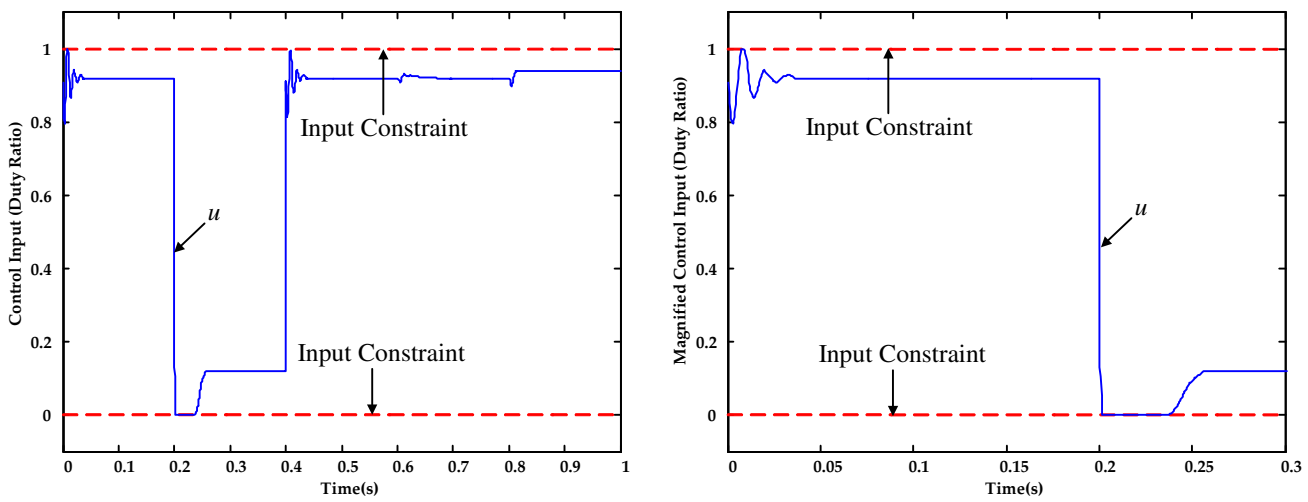

Figure 6. The saturated control input behavior with its magnified version during $0 \sim 0.3 \mathrm{~s}$.

\section{Conclusions}

In this study, we propose an offset-free global tracking state-feedback control algorithm for the controllable and strictly stable LTI systems, considering the input constraints. The proposed controller is very simple in such a way that it feedbacks the estimated tracking error and steady state control using an estimated state of the DOB; any online numerical optimization process is not included. It is shown that the proposed controller ensures the global output tracking property in the presence of input constraints, thanks to the invariance property of the projection operator, and that the steady state errors in the output can be removed if some rank condition is satisfied. Furthermore, by performing the realistic simulations using the DC/DC buck converter, it is also verified that the proposed control method can be an effective solution to the global output tracking problem of the LTI systems under the input constraints. In the future, we plan to apply the proposed method to the open-loop unstable systems with the experimental verification using a prototype hardware, such as power converters and motor drives.

Author Contributions: Conceptualization and methodology, S.-K.K.; software, validation, formal analysis, investigation, writing - original draft preparation, and writing - review and editing, K.C.; resources, supervision, project administration, and funding acquisition, D.S.K. All authors have read and agreed to the published version of the manuscript

Funding: This work was supported in part by by a grant 17TLRP-C135446-01 from the Transportation \& Logistics Research Program (TLRP) funded by the Ministry of Land, Infrastructure, and Transport of the Korean Government, and was supported in part by Basic Science Research Program through the National Research Foundation of Korea(NRF) funded by the Ministry of Education(2018R1A6A1A03026005).

Conflicts of Interest: The authors declare no conflict of interest.

\section{References}

1. Danayiyen, Y.; Lee, K.; Choi, M.; Lee, Y.I. Model Predictive Control of Uninterruptible Power Supply with Robust Disturbance Observer. Energies 2019, 12, 2871. [CrossRef]

2. Krishnan Prakash, A.; Zhang, K.; Gupta, P.; Blum, D.; Marshall, M.; Fierro, G.; Alstone, P.; Zoellick, J.; Brown, R.; Pritoni, M. Solar+ Optimizer: A Model Predictive Control Optimization Platform for Grid Responsive Building Microgrids. Energies 2020, 13, 3093. [CrossRef]

3. Ferro, G.; Robba, M.; Sacile, R. A Model Predictive Control Strategy for Distribution Grids: Voltage and Frequency Regulation for Islanded Mode Operation. Energies 2020, 13, 2637. [CrossRef]

4. Palmieri, A.; Rosini, A.; Procopio, R.; Bonfiglio, A. An MPC-Sliding Mode Cascaded Control Architecture for PV Grid-Feeding Inverters. Energies 2020, 13, 2326. [CrossRef]

5. Sasaki, Y.; Tsubakino, D. Designs of Feedback Controllers for Fluid Flows Based on Model Predictive Control and Regression Analysis. Energies 2020, 13, 1325. [CrossRef]

6. Lim, J.; Kim, J.S.; Lee, Y. Robust tracking model predictive control for input-constrained uncertain linear time invariant systems. Int. J. Control 2013, 87, 120-130. [CrossRef] 
7. Maedera, U.; Borrelli, F.; Morari, M. Linear offset-free Model Predictive Control. Automatica 2009, 45, $2214-2222$. [CrossRef]

8. Limon, D.; Alvarado, I.; Alamo, T.; Camacho, E.F. MPC for tracking piecewise constant references for constrained linear systems. Automatica 2008, 44, 2382-2387. [CrossRef]

9. Chisci, L.; Zappa, G. Dual mode predictive tracking of piecewise constant references for constrained linear systems. Int. J. Control 2003, 76, 61-72. [CrossRef]

10. Pannocchia, G.; Kerrigan, E.C. Offset-free Receding Horizon Control of Constrained Linear Systems. AIChE J. 2005, 51, 3134-3146. [CrossRef]

11. Sussmanna, H.J.; Sontag, E.D.; Yang, Y. A general result on the stabilization of linear systems using bounded controls. IEEE Trans. Autom. Control 1994, 39, 2411-2425. [CrossRef]

12. Suarez, R.; Alvarez-Ramirez, J.; Solis-Daun, J. Linear systems with bounded inputs: Global stabilization with eigenvalue placement. Int. J. Robust Nonlin. Control 1997, 7, 835-845. [CrossRef]

13. LIN, Z. Global Control of Linear Systems with Saturating Actuators. Automatica 1998, 34, 897-905. [CrossRef]

14. Grimm, G.; Hatfield, J.; Postlethwaite, I.; , A.R.T.; Turner, M.C.; Zaccarian, L. Antiwindup for Stable Linear Systems With Input Saturation: An LMI-Based Synthesis. IEEE Trans. Autom. Control 2003, 48, 1509-1525. [CrossRef]

15. Kim, J.S.; Yoon, T.W.; Jadbabaie, A.; Persis, C.D. Input-to-state stable finite horizon MPC for neutrally stable linear discrete-time systems with input constraints. Syst. Control Lett. 2006, 55, 293-303. [CrossRef]

16. Wanga, X.; Saberi, A.; Stoorvogel, A.A. Stabilization of linear system with input saturation and unknown constant delays. Automatica 2013, 49, 3632-3640. [CrossRef]

17. Blanchini, F. Set invariance in control. Automatica 1999, 35, 1747-1767. [CrossRef]

18. Hu, T.; Lin, Z. Composite Quadratic Lyapunov Functions for Constrained Control Systems. IEEE Trans. Autom. Control 2003, 48, 440-450.

19. Gayaka, S.; Lua, L.; Yao, B. Global stabilization of a chain of integrators with input saturation and disturbances: A new approach. Automatica 2012, 48, 1389-1396. [CrossRef]

20. Boyd, S.; Vandenberghe, L. Convex Optimization; Cambridge University Press: Cambridge, UK, 2004.

21. Krstic, M.; Kanellakopoulos, L.; Kokotovic, P. Nonlinear and Adaptive Control Design; Wiley-Interscience: Hoboken, NJ, USA, 1995.

22. Rudin, W. Principles of Mathematical Analysis; McGRAW-HILL: New York, NY, USA, 1976.

23. Kassakian, J.C.; Schlecht, M.; Verghese, G.C. Principles of Power Electronics; Addison-Wesley: Boston, MA, USA, 1991.

24. Kim, S.K.; Park, C.R.; Kim, J.S.; Lee, Y.I. A Stabilizing Model Predictive Controller for Voltage Regulation of a DC/DC Boost Converter. IEEE Trans. Control Syst. Technol. 2014, 22, 2016-2023. [CrossRef]

25. Cortes, P.; Ortiz, G.; Yuz, J.I.; Rodriguez, J.; Vazquez, S.; Franquelo, L.G. Model Predictive Control of an Inverter With Output LC Filter for UPS Applications. IEEE Trans. Ind. Electron. 2009, 56, 1875-1883. [CrossRef]

(C) 2020 by the authors. Licensee MDPI, Basel, Switzerland. This article is an open access article distributed under the terms and conditions of the Creative Commons Attribution (CC BY) license (http://creativecommons.org/licenses/by/4.0/). 